\title{
POLICITEMIA PÓS-TRANSPLANTE: INCIDÊNCIA, FATORES DE RISCO E PROGNÓSTICO
}

\author{
Post-Transplant Polycythemia: Incidence, risk factors and prognosis
}

André Luis Bastos Sousa, Marcos Vinícius de Sousa, Leonardo Figueiredo Camargo, Gabriel Giollo Rivelli, Marilda Mazzali

\section{RESUMO}

Introdução: Avaliar a incidência de policitemia (PTxP) em transplantados renais no primeiro ano pós-transplante. Identificar fatores de risco e implicações prognósticas. Métodos: Estudo retrospectivo, observacional. Critérios de inclusão: receptores de transplante renal entre janeiro e dezembro de $2010 \mathrm{com}$ idade $>18$ anos, acompanhamento pós $t x>$ seis meses. Exclusão: DPOC, tabagismo ativo e eritrocitose secundária. Para análise, os pacientes foram divididos em dois grupos: PTxP: $\mathrm{Hb} \geq 18 \mathrm{~g} / \mathrm{L}$ ou htc $\geq 51 \%$ (homens) ou $\mathrm{Hb} \geq 17 \mathrm{~g} / \mathrm{L}$ ou Htc $\geq 50 \%$ (mulheres). PTxP grave foi definida como $\mathrm{hb}>18,5 \mathrm{~g} / \mathrm{L}$ ou $\mathrm{htc}>55 \%$ e/ou necessidade de sangria terapêutica. Grupo controle (CTL): $\mathrm{Hb}<18 \mathrm{~g} / \mathrm{L}$ ou htc $<51 \%$ (homens) e $\mathrm{hb}<17 \mathrm{~g} / \mathrm{L}$ ou ht $<50 \%$ (mulheres). Parâmetros avaliados: dados demográficos, eventos tromboembólicos, hemoglobina, hematócrito e creatinina sérica, terapêutica e evolução. Resultados: 122 pacientes, idade $47 \pm 12$ anos, maioria homens $(63,1 \%)$ e receptores de rim de doador falecido (95\%) preencheram

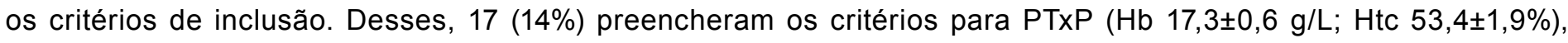
diagnosticada $9 \pm 5$ meses pós-transplante. Seis pacientes classificados como PTxP grave não apresentaram fenômeno tromboembólico grave e/ou necessidade de flebotomia. Cerca de $62 \%$ dos pacientes apresentavam creatinina $<1,6$ $\mathrm{g} / \mathrm{L}$ após seis meses de acompanhamento. O tratamento foi realizado com iECA e/ou aminofilina em 12/17 pacientes, com resposta completa em oito casos. Dos cinco pacientes não tratados, quatro evoluíram com remissão espontânea completa. Os grupos PTxP e CTL eram comparáveis em parâmetros demográficos, pressão arterial, função renal, diabetes ou tabagismo pré-transplante e/ou terapia imunossupressora. O grupo PTxP evoluiu com recuperação mais rápida dos parâmetros hematimétricos que o CTL. Não houve diferença significativa entre grupos para os desfechos de perda de enxerto e morte em três anos, mas houve tendência à perda de enxerto mais precoce no grupo CTL. Conclusão: A incidência de policitemia foi de $14 \%$, menor do que a reportada em nossa série histórica, de $33 \%$. Níveis de hemoglobina próximos ao normal no $1^{\circ}$ mês pós-transplante foram marcadores precoces de PTxP.

Keywords: Transplante; Policitemia; Resultado de Tratamento.

Instituição:

Programa de Transplante Renal - Disciplina de Nefrologia Departamento de Clínica Médica - Faculdade de Ciências Médicas Universidade Estadual de Campinas- DCM/FCM/ UNICAMP Campinas/SP - Brasil

\section{Correspondência:}

Profa. Dra. Marilda Mazzali

Disciplina de Nefrologia, Departamento de Clínica Médica

Faculdade de Ciências Médicas - UNICAMP - Rua Tessália Vieira de Camargo 126- Cidade Universitária Zeferino Vaz

13086-970- Campinas- São Paulo- Brazil

fone: (55) 19-35218204 - fax: (55) 19-3521-8206

email: marildamazzali@gmail.com

Recebido em: 13/07/2014

Aceito em: 23/09/2014

\section{INTRODUÇÃO}

Policitemia é definida como hematócrito (ht) superior a $51 \%$ ou hemoglobina $(\mathrm{hb}) \geq 18 \mathrm{~g} / \mathrm{dL}$ para homens ou hematócrito $>50 \%(\mathrm{Hb} \geq 17 \mathrm{~g} / \mathrm{dL})$ para mulheres após o sexto mês de transplante renal, desde que excluídas outras causas potenciais de eritrocitose., ${ }^{1,2}$ É uma complicação que acomete muitos receptores de transplante renal, apesar de apresentar redução progressiva de incidência na última década, associada ao aumento do uso de imunossupressores antiproliferativos, de bloqueadores do sistema renina angiotensina aldosterona, iECA (inibidores do enzima conversora da angiotensina) e BRAs (bloqueadores dos receptores de angiotensina)..$^{2,3}$ 
Cerca de $65 \%$ dos casos de policitemia pós-transplante (PTxP) ocorrem durante o primeiro ano pós-transplante e $86 \%$ nos primeiros dois anos, ${ }^{4,5}$ geralmente em pacientes com função renal preservada do enxerto. ${ }^{4} \mathrm{O}$ pico nos níveis de hemoglobina tende a ocorrer entre 16 e 20 meses após o transplante ${ }^{2,5}$ e cerca de $25 \%$ dos pacientes podem evoluir com remissão espontânea. ${ }^{6}$

Fatores de risco para PTxP incluem o gênero masculino, ausência de episódios de rejeição aguda, função renal preservada do enxerto, persistência dos rins nativos, diabetes e doença renal policística como causa de doença renal primária, estenose da artéria renal do enxerto, controle de hiperparatireoidismo e toxicidade por ciclosporina. ${ }^{4,6-8} \mathrm{O}$ diagnóstico diferencial inclui causas secundárias para aumento da viscosidade sanguínea, como uso de diuréticos de alça, doença pulmonar crônica e hipovolemia. ${ }^{6}$

A patogênese da PTxP é multifatorial e permanece controversa. ${ }^{6,7}$ Possíveis mecanismos envolvem (a) aumento da produção de eritropoetina (EPO) pelos rins nativos; ${ }^{3}$ (b) aumento da sensibilidade para EPO e aumento da eritropoese mediada por fatores de crescimento como IGF-1 (insulin-like growth factors) e GSCSF (growth stem cells soluble factor); ${ }^{8}$ (c) proliferação de precursores eritroides mediado por angiotensina ${ }^{9}$ e (d) efeito de andrógenos endógenos, que estimulariam progenitores eritroides e explicariam a elevada incidência de PTxP em homens. ${ }^{6}$

A apresentação clínica da PTxP inclui tonturas, cefaleia, letargia e adinamina, associadas ao aumento da viscosidade sanguínea. Cerca de $15 \%$ dos pacientes desenvolvem eventos tromboembólicos arteriais ou venosos, variando de trombose periférica a tromboembolismo pulmonar. ${ }^{4,6}$

Com o objetivo de avaliar a incidência, fatores de risco e implicações na sobrevida de paciente, analisamos de forma retrospectiva um grupo de receptores de transplante renal durante três anos após o transplante.

\section{PACIENTES E MÉTODOS}

Estudo descritivo, retrospectivo, observacional incluindo receptores de transplante renal durante os primeiros três anos após o transplante.

Critérios de Inclusão: (a) receptores de transplante renal isolado entre janeiro e dezembro de 2010; (b) idade $\geq 18$ anos na ocasião do transplante e (c) acompanhamento pós-transplante $\geq$ seis meses. Foram considerados como critérios de exclusão: (a) perda de paciente e enxerto nos primeiros seis meses pós-transplante; (b) diagnóstico de DPOC (doença pulmonar obstrutiva crônica), tabagismo ativo e/ou policitemia secundária (c) uso crônico de diuréticos de alça. Os pacientes elegíveis foram divididos em dois grupos, de acordo com os níveis de hematócrito nos grupos de Policitemia (PTxP) e controle normal (CTL), e acompanhados por um período de três anos.

Policitemia pós-transplante foi definida como $\mathrm{hb} \geq 18 \mathrm{~g} /$ $\mathrm{dL}$ e hematócrito $\geq 51 \%$ para homens e $\mathrm{hb} \geq 17 \mathrm{~d} / \mathrm{dL}$ e ht $\geq 50 \%$ para mulheres. Eritrocitose grave foi definida como hb $>18.5 \mathrm{~g} / \mathrm{dL}$ ou $\mathrm{Ht}>55 \%$ e/ou necessidade de sangrias terapêuticas regulares.

Dados clínicos e laboratoriais foram coletados a partir dos prontuários médicos e do banco de dados do programa de transplante renal e incluíram: (a) dados demográficos: idade, gênero, etiologia da doença renal primária, tipo de doador (vivo ou falecido); (b) Eventos associados com PTxP, incluindo tromboembolismo, hipertensão arterial, diabetes e rejeição aguda comprovada por biopsia. (c) fatores de risco para PTxP: estenose de artéria renal do enxerto, uso de diuréticos de alça, tabagismo e imunossupressores; (d) parâmetros laboratoriais: hemoglobina, hematócrito, creatinina sérica e (e) terapia para controle de PTxP: medicamentos e doses. Os dados foram coletados nos períodos basal e a intervalos de três meses, por três anos.

Os tesultados foram apresentados como média \pm desvio padrão. As variáveis categóricas foram analisadas por teste exato de Fisher, enquanto que as variáveis contínuas foram analisadas pelo teste $t$ de Student. A significância estatística foi considerada em <0,05.

\section{RESULTADOS}

Entre janeiro e dezembro de 2010, foram realizados 136 transplantes renais em pacientes $>18$ anos, dos quais 14 foram excluídos por perda do enxerto ou óbito com rim funcionante, nos primeiros seis meses pós-transplante. $O$ grupo de estudo foi composto de 122 pacientes, a maioria do gênero masculino ( $n=77,63,1 \%)$, com idade média de $47 \pm 12$ anos, sendo $95 \%$ receptores de rim de doador falecido. As causas da doença renal primária foram hipertensão arterial sistêmica (27\%), glomerulonefrite crônica $(18 \%)$, diabetes $(13,1 \%)$, pielonefrite crônica (9\%), doença renal policística (APKD, 8,1\%) e etiologia indeterminada (17,2\%). Após o transplante, 95\% dos pacientes apresentavam hipertensão arterial e 33,6\% diabetes. Rejeição aguda comprovada por biopsia foi observada em $13,6 \%$ dos pacientes. A sobrevida de enxerto em três anos foi de $88,8 \%$ e as principais causas de perda de enxerto foram morte com rim funcionante $(46,1 \%)$, disfunção crônica do enxerto $(23,3 \%)$, rejeição aguda (15,3\%), e nefropatia pelo Poliomavírus humano 
(15,3\%). A taxa de mortalidade em três anos foi de $5,5 \%$, de etiologia infecciosa $(60 \%)$ ou cardiovascular (33\%).

Dezoito pacientes preencheram os critérios laboratoriais para policitemia com incidência de $14 \%$, diagnosticado em média $9 \pm 5$ meses após o transplante. No diagnóstico, a hemoglobina média foi de 17,3 $\pm 0,6 \mathrm{~g} / \mathrm{dL}$ com hematócrito de 53,4 $\pm 1,9 \%$.

Os grupos PTxP e de controle eram comparáveis em relação à idade, ao gênero, tipo de doador (vivo ou falecido), à doença renal primária, etiologia da doença renal primária, história de tabagismo ou diabetes prétransplante, pressão arterial, função renal e incidência de rejeição aguda confirmada por biopsia. [Tabela 1].

Tabela 1 -Parâmetros clínicos e demográficos dos grupos estudados.

\begin{tabular}{lcc}
\hline \multicolumn{1}{c}{ Parâmetro } & PTxP & Controle \\
\hline Número de pacientes & 17 & 104 \\
Idade (anos) & $44,4 \pm 11,4$ & $47,3 \pm 11,5$ \\
Gênero (masculino:feminino) & $14: 3$ & $63: 41$ \\
Doador (vivo:falecido) & $2: 15$ & $4: 100$ \\
Tromboembolismo (\%) & 0 & $1(0,9 \%)$ \\
Tabagismo (\%) & 41,1 & $25,0 *$ \\
Hipertensão arterial (\%) & 100 & 94,2 \\
Diabetes pré-transplante (\%) & 23,5 & 35,5 \\
Diabetes pós-transplante (\%) & 0 & 13,4 \\
Rejeição aguda em biopsia (\%) & 5,8 & 15,3 \\
Pressão arterial sistólica (mmHg) & & \\
PAS transplante & $140 \pm 15$ & $143 \pm 21$ \\
PAS M1 & $121 \pm 19$ & $126 \pm 17$ \\
PAS M3 & $130 \pm 14$ & $131 \pm 20$ \\
PAS M6 & $127 \pm 23$ & $133 \pm 22$ \\
Pressão arterial diastólica (mmHg) & & \\
PAD transplante & $82 \pm 9$ & $80 \pm 12$ \\
PAD M1 & $78 \pm 16$ & $78 \pm 12$ \\
PAD M3 & $79 \pm 11$ & $80 \pm 11$ \\
PAD M6 & $81 \pm 13$ & $82 \pm 11$ \\
\hline
\end{tabular}

${ }^{*} \mathrm{P}<0.05$ Versus Controle

Desde o primeiro mês pós-transplante, os níveis de hematócrito e hemoglobina do grupo PTxP foram significativamente superiores aos do grupo de controle, mostrando recuperação mais rápida da anemia, independente da função renal do enxerto. [Tabela 2]

A análise da terapia imunossupressora mostrou tendência à maior utilização de ciclosporina no grupo $\operatorname{PTxP}(17,4$ verus $3,8 \%$, PTxP versus controle, $p=0,05)$. Para os demais agentes imunossupressores, não observamos diferença entre os grupos.

No grupo com diagnóstico de policitemia, a função renal permaneceu estável durante todo o período de acompanhamento, com creatinina média de 1,4 \pm $0,5 \mathrm{mg} / \mathrm{dL}$. A terapia para PTxP foi necessária em 12 pacientes, com bloqueio do sistema renina angiotensina aldosterona isolado ou associado a aminofilina, resultando em controle do hematócrito em oito (67\%) pacientes. A maioria dos pacientes que não recebeu terapia farmacológica apresentou remissão espontânea da PTxP.

Tabela 2 -Valores do RMS com e sem a utilização de pressão positiva expressos em média e desvio padrão

\begin{tabular}{ccccccc}
\hline & \multicolumn{2}{c}{ Hemoglobina } & \multicolumn{2}{c}{ Hematócrito } & \multicolumn{2}{c}{ Creatinina } \\
\hline & PTxP & Controle & PTxP & Controle & PTxP & Controle \\
TX & $11.7 \pm 2.2$ & $11.8 \pm 1.7$ & $35.7 \pm 6.5$ & $36.2 \pm 5.4$ & $9.6 \pm 4.1$ & $8.4 \pm 2.6$ \\
M1 & $11.8 \pm 1.7^{*}$ & $10.3 \pm 1.5$ & $36.2 \pm 5.0^{*}$ & $31.7 \pm 4.7$ & $2.4 \pm 1.9$ & $1.8 \pm 0.9^{*}$ \\
M3 & $14.1 \pm 1.6^{*}$ & $11.8 \pm 1.8$ & $43.6 \pm 4.2^{*}$ & $37.0 \pm 5.7$ & $1.3 \pm 0.2$ & $1.5 \pm 0.6$ \\
M6 & $16.6 \pm 1.3^{*}$ & $13.0 \pm 1.6$ & $51.5 \pm 4.1^{*}$ & $40.5 \pm 4.9$ & $1.3 \pm 0.2$ & $1.5 \pm 0.6$ \\
\hline
\end{tabular}

${ }^{*} \mathrm{P}<0.05$ Versus Controle

A sobrevida do paciente e do enxerto também foram comparáveis entre os grupos durante o acompanhamento de três anos. No grupo PTxP ocorreram duas perdas de enxerto causadas por óbito com rim funcionante $(n=1)$ e nefropatia pelo Poliomavírus $(n=1)$. No grupo de controle, 11 enxertos foram perdidos, por morte com rim funcionante $(n=5)$, rejeição aguda $(n=3)$ e disfunção crônica do enxerto $(n=3)$. Observamos tendência à perda mais precoce do enxerto no grupo de controle $(19,7$ versus 24 meses, controle versus PTxP, $p=0,06)$, assim como para morte do paciente $(12,4$ versus 23,0 meses, controle versus PTxP, $p<0,05)$. Entretanto, esses dados devem ser analisados com cautela, uma vez que a rejeição aguda confirmada por biopsia ocorreu apenas no grupo de controle, o que pode ter impacto negativo na sobrevida do enxerto.

\section{DISCUSSÃO}

Nesta série de 122 casos, a incidência de policitemia pós-transplante foi de 14\%, comparável a outros estudos, com incidência variando de 8 a $15 \%,{ }^{2.6}$ dependendo do critério diagnóstico utilizado. Em estudo anterior do nosso grupo com 333 pacientes transplantados entre janeiro de 1984 e dezembro de 1993, a incidência de PTxP foi de $33 \%$, significativamente superior à presente série. ${ }^{10}$ Essa diferença pode ser atribuída ao uso de diferentes protocolos de imunossupressão, utilizando drogas com potencial antiproliferativo mais potente, como derivados do ácido micofenólico ou inibidores da mTOR, comparado ao grupo anterior, com imunossupressão baseada em azatioprina e ciclosporina. ${ }^{2}$ Também deve ser considerado que no início da década de 1990, o uso 
de bloqueio do sistema renina angiotensina aldosterona era controverso e evitado por muitos grupos. ${ }^{11,12}$

O tempo médio para diagnóstico de PTxP foi de 10 meses, consistente com estudos anteriores, onde $65 \%$ dos casos ocorreram durante o primeiro ano após o transplante..$^{4-6}$ Apesar da elevada prevalência de homens no grupo PTxP (65\%), não pudemos comprovar, nesta série, um risco aumentado de policitemia associada ao gênero masculino. ${ }^{2,4,6}$ Outros fatores de risco potenciais para PTxP, como função renal do enxerto ou doença renal policística como causa de DRC também não diferiram de forma significativa entre os grupos. Os níveis de hemoglobina próximos ao valor da normalidade ao final do primeiro mês de transplante mostram a recuperação rápida da anemia no pós-operatório, podendo ser considerado fator de risco para desenvolvimento de PTxP nesses pacientes.

Apesar do risco aumentado de hiperviscosidade associado à policitemia, predispondo ao desenvolvimento de fenômenos tromboembólicos, não observamos episódios de tromboses vasculares nos pacientes do grupo PTxP.13 Esse achado pode ser explicado pelo pequeno número de pacientes com $\mathrm{PTXP}$, pela resolução espontânea em alguns casos e também pela intervenção terapêutica precoce, com controle do hematócrito e da hiperviscosidade, com redução do risco de complicações cardiovasculares. . $^{2,12}$

Nos pacientes que desenvolveram PTxP, os níveis de hematócrito e hemoglobina mais elevados já no primeiro mês pós-transplante e persistentes no primeiro trimestre podem ser considerados como um marcador precoce de policitemia, sugerindo o início precoce de inibidores da enzima conversora de angiotensina (iECA) ou bloqueadores do receptor de angiotensina (BRA) como medicamentos de escolha para tratamento da hipertensão arterial e da PTxP, pelo seu efeito inibitório sobre a eritropoiese..$^{10,12,14}$ A imunossupressão com ciclosporina foi mais frequente no grupo PTxP, porém o uso de ciclosporina foi inferior a $25 \%$ na população geral do estudo. Estudos anteriores mostraram dados controversos ao considerar a ciclosporina como fator de risco para $\mathrm{PTxP}$, onde o fator isquêmico induzido pela toxicidade desse medicamento poderia estimular a produção de eritropoetina pelo enxerto. ${ }^{2,4,6}$ Quando comparamos a incidência de PTXP nessa série com os dados históricos do nosso grupo, quando a ciclosporina era a imunossupressão padrão, a diferença de incidência de $14 \%$ para $33 \%$ pode sugerir a ciclosporina como fator de risco. Entretanto, devemos considerar que na série histórica a imunossupressão baseava-se em azatioprina, que apresenta efeito inibitório medular para série eritrocitária inferior ao dos derivados do ácido micofenólico e dos inibidores da mTOR, mais associados ao desenvolvimento de anemia pós-transplante..$^{10,13,15}$

Após três anos de acompanhamento, a sobrevida de paciente e enxerto foram comparáveis entre os grupos, com tendência à falência de enxerto mais precoce no grupo de controle. Entretanto, $25 \%$ das perdas de enxerto no grupo de controle ocorreram por rejeição aguda, o que pode ter afetado negativamente a análise, uma vez que no grupo PTxP não observamos nenhum episódio de rejeição aguda confirmada por biopsia.

\section{CONCLUSÃO}

$\mathrm{Na}$ presente série, a ausência de eventos tromboembólicos no grupo PTxP pode ser consequência, tanto do pequeno número de pacientes, como da resolução espontânea de alguns casos e também da intervenção precoce, controlando assim esse fator de risco cardiovascular. Entretanto, complicações associadas à síndrome de hiperviscosidade devem ser consideradas como risco aumentado para esse grupo de pacientes, levando à necessidade de controle estrito dos níveis de hematócrito e da hemoglobina.

Este artigo apresenta algumas limitações, especialmente por seu desenho retrospectivo e observacional. $O$ número de pacientes com PTxP foi pequeno e pode ter influenciado a análise estatística. Entretanto, a intervenção precoce nos casos de PTxP pode estar associada à baixa incidência de complicações tanto cardiovasculares como de eventos tromboembólicos nesta série. 


\section{ABSTRACT}

Purpose: To assess the incidence of post transplant polycythemia (PTxP) in the first year post transplant in a series of renal transplant recipients and identifying risk factors and prognosis. Methods: Retrospective, observational study. Criteria included: renal transplant from January to December 2010, age $>18$ years at transplant, post-transplant follow-up $>6$ months. Criteria of exclusion: obstructive lung disease, active smoking and secondary erythrocytosis. For the analysis, patients were divided in 2 groups: Polycythemia (PTxP) Hb $\geq 18 \mathrm{~g} / \mathrm{L}$ or htc $\geq 51 \%$ (male) or Hb $\geq 17 \mathrm{~g} / \mathrm{L}$ or $\mathrm{Htc} \geq 50 \%$ (female). Severe PTxP was defined as $\mathrm{hb}>18,5 \mathrm{~g} / \mathrm{L}$ or htc $>55 \%$ and/or need of phlebotomies. Control group (CTL): $\mathrm{Hb}<18 \mathrm{~g} / \mathrm{L}$ or htc $<51 \%$ (male) and $\mathrm{hb}<17 \mathrm{~g} / \mathrm{L}$ or $\mathrm{ht}<50 \%$ (female). Data analyzed included demographic information, thromboembolism, laboratory data such as hemoglobin, hematocrit and serum creatinine, therapy and adverse events. Results: 122 patients, $47 \pm 12$ years old, majority male $(63,1 \%)$ and recipients of kidneys from deceased donors (95\%) fulfilled the inclusion criteria. Seventeen patients (14\%) were classified as PTxP (Hb 17,3 $\pm 0,6 \mathrm{~g} / \mathrm{L}$; Htc $53,4 \pm 1,9 \%$ ), diagnosed $9 \pm 5$ months after transplant. Six patients classified as bearers of severe PTxP were free of thromboembolic events or need for phlebotomies during the follow-up. Up to $62 \%$ patients presented normal renal function (serum creatinine $<1,6 \mathrm{mg} / \mathrm{dL}$ ) after the 6 months follow up. PTxP therapy included ACEi or amminophylline in 12/17 patients, with complete remission in 8 . From 5 untreated patients, 4 had complete spontaneous remission. PTxP and CTL groups had comparable demographic data, blood pressure, renal function and were under similar immunossupressive therapy. In addition, the incidence of diabetes and smoking was similar between groups. In the PTxP group, anemia recovered earlier than in CTL group, despite of presenting similar renal function. The 3-year graft and patient survival was similar between groups with a trend to earlier graft loss in CTL group. Conclusion: The incidence of PTxP in this series was 14\%, lower than previous report from our group of $33 \%$. The near normal hemoglobin level range within the first month post transplant was the only marker for the PTxP risk in this series.

Keywords: Transplantation; Polycythemia; Treatment Outcome.

\section{REFERÊNCIAS}

1. Kasiske BL, Vazquez MA, Harmon WE, Brown RS, Danovitch GM, Gaston RS et al. Recommendations for the outpatient surveillance of renal transplant recipients. American Society of Transplantation. J Am Soc Nephrol 2000;11(Suppl 15):S1-86.

2. Kiberd BA. Post-transplant erythrocytosis: a disappearing phenomenon? Clin Transplant 2009; 23(6):800-6.

3. Abbrecht $\mathrm{PH}$, Greene JA Jr. Serum erytropoeitin after renal homotransplantation. J Int Med 1966;65(5):908-21.

4. Einollahi B, Lessan-Pezeshki M, Nafar M, Pour-Reza-Gholi F, Firouzan A, Farhangi $F$ et al: Erythrocytosis after renal transplantation: Review of 101 Cases. Transplant Proceed 2005;37(7):3101-2.

5. Kolonko A, Pinocy-Mandok J, Kocierz M, Kujawa-Szewieczek $\mathrm{A}$, Chudek J, Malyszko J et al. Anemia and erythrocytosis after kidney transplantation: A 5-year graft function and survival analysis. Transplant Proceed 2009;41(8):3046-51.

6. Vlahakos DV, Marathias KP, Kosmas EN. Posttransplant erythrocytosis. Kidney Int 2003;63(4):1187-94.

7. Aeberhard JM, Schneider PA, Vallotton MB, Kurtz A, Leski $M$. Multiple site estimates of erythropoietin and renin in polycythemic kidney transplant patients. Transplantation 1990;50(4):613-6.

8. Kiykim AA, Genctoy G, Horoz M, Tiftik NE, Gok E, Altun $B$ et al. Serum stem cell factor level in renal transplant recipients with posttransplant erythrocytosis. Artif Organs. 2009;33(12):1086-90.
9. Gossmann J, Burkhardt R, Harder S, Lenz T, Sedlmeyer A, Klinkhardt $U$, Geiger $\mathrm{H}$ et al. Angiotensin II infusion increases plasma erythropoietin levels via an angiotensin II type 1 receptor-dependent pathway. Kidney Int 2001;60(1):83-6.

10. Mazzali M, Filho GA: Use of aminophylline and enalapril in posttransplant polycythemia. Transplantation 1998;65(11):1461-4.

11. Hiremath S, Fergusson D, Doucette S, Mulay AV, Knoll GA. Renin angiotensin system blockade in kidney transplantation: a systematic review of the evidence. Am J Transplant 2007;7(10):2350-60.

12. Zhu X, Chen J, Han E, Cheng M, Xu L, Zhang L et al. Efficacy and safety of losartan in treatment of hyperuricemia and posttransplantation erythrocytosis: results of a prospective, open, randomized, case-control study. Transplant Proceed 2009;41(9):3736-42.

13. Marinella MA. Hematologic abnormalities following renal transplantation. Int Urol Nephrol 2010; 42(1): 151-64.

14. Bakris GL, Sauter ER, Hussey JL, Fisher JW, Gaber AO, Winsett R. Effects of theophylline on erythropoietin production in normal subjects and in patients with erythrocytosis after renal transplantation. $\mathrm{N}$ Engl J Med 1990;323(2):86-90.

15. Kasiske BL, Gaston RS, Gourishankar S, Halloran PH, Matas AJ, Jeffery $\mathrm{J}$ et al. Long-term deterioration of kidney allograft function. Am J Transplant 2005;5(6):140514. 